\title{
The risk of cold temperature: an important aspect of the determination of morning blood pressure surge
}

\author{
Yuichiro Yano and Kazuomi Kario \\ Hypertension Research (2011) 34, 36-38; doi:10.1038/hr.2010.203; published online 28 October 2010
}

$I_{d}^{t}$ t has become accepted that acute myocardial infarction, sudden cardiac death and stroke can be triggered by stressors such as heavy physical exertion, severe emotional stress and environmental stress. ${ }^{1}$ The mechanisms underlying these associations are not fully understood, but the blood pressure (BP) variability that can be introduced by such triggers is thought to be one of the important factors. ${ }^{2}$

BP is a highly variable physiological trait, the fluctuation of which is a complex interaction between external environmental stimuli and the responses of individual cardiovascular systems. ${ }^{3-7}$ From the view point of clinical management, BP variability consists of at least two types of phenomena: longterm fluctuations, such as day-night, day-today, weekly (for example, Monday BP surge) and seasonal changes, and short-term BP variations that occur within the daytime and nighttime periods (Figure 1). Several studies, but not all, have demonstrated that both short-term BP variability and long-term BP variability themselves are significantly associated with the target-organ damage and with future cardiovascular events independently of the 24-h BP levels. ${ }^{3-7}$ Intriguingly, certain reports have suggested that the peak of the incidence of cardiovascular diseases (CVDs) is likely to parallel the time with a greater extent of the BP variations. ${ }^{1,8,9}$ For example, with respect to diurnal variation, most studies have shown an increased incidence of acute myocardial infarction, sudden cardiac death and stroke during the morning periods after waking up, and this variation

Y Yano is at the Divisions of Community and Family Medicine, University of Miyazaki, Miyazaki, Japan and Dr K Kario and Y Yano are at the Division of Cardiovascular Medicine, Department of Medicine, Jichi Medical University School of Medicine, Tochigi, Japan.

E-mail: kkario@jichi.ac.jp appears to be closely associated with the diurnal variation of BP (that is, morning BP increase). 9,10

In our prospective cohort study ( 3.5 years) of 519 elderly Japanese patients with hypertension, ${ }^{9}$ an excessive increase in morning BP on awakening (sleep-through morning BP surge: sleep-through MS), which was calculated using the difference between the mean systolic BP during the $2 \mathrm{~h}$ after waking up and the average of three readings centered on the lowest-sleep BP ( $\geqslant 55 \mathrm{~mm} \mathrm{Hg:} \mathrm{top} \mathrm{decile} \mathrm{of}$ the population), was found to be associated with an increased risk of stroke compared with a nonsurge group (19 vs. $7.3 \%$, respectively; $P=0.004$ ), and this association was independent of the ambulatory 24-h BP level. In that study, we found that seven (78\%) of the nine stroke events in the MS group occurred in the morning period $(0600 \mathrm{~h}$ to noon), whereas $11(41 \%)$ of the 27 events in the non-MS group occurred during this period $\left(x^{2}=3.70, P=0.05\right)$. This means that an excessive morning BP increase might trigger strokes through some hemodynamic mechanism, such as increased shear stress on the atherosclerotic cerebral vessels, although there remain several other risk factors that change during the morning hours, including sympathetic nervous activity (particularly sympathetic adrenergic activity) and other related acute risk factors such as platelet hyperactivity, hypercoagulability and hypofibrinolysis, blood viscosity and increased vascular spasm.

A seasonal variation in the incidence of acute myocardial infarction, sudden cardiac death and stroke is also consistently described, with winter peak up to $60 \%$ greater than the summer nadir. ${ }^{1,11}$ The mechanisms underlying this variation are not fully understood, although cold ambient temperature could cause sympathetic nervous system activity that in turn could cause increased heart rate and BP, a hypercoagulable state, proinflammatory changes and abnormalities in serum lipids and glucose. ${ }^{1,12,13}$ Several studies have demonstrated that BP is subject to seasonal influences and is lower in hot than in cold months. ${ }^{13-15}$ This influence is not limited to office measurements but affects daily values (that is, home BP and ambulatory BP) as well. ${ }^{15}$ Moreover, Modesti et al. ${ }^{16}$ recently examined the association between air temperature and ambulatory BP profile: they showed that cold temperature was significantly associated with excessive sleep-through MS in elderly persons, even when they were treated with a higher number of antihypertensive drugs in cold weather. This cold temperature-augmented morning BP increase may partly account for the increased number of CVDs on cold mornings during the winter season. ${ }^{17}$

In the current issue of Hypertension Research, Murakami et al. ${ }^{18}$ make an important contribution toward our understanding of the association between cold temperature and morning BP increase. In this cross-sectional survey of 158 subjects who had performed 24-h ambulatory BP monitoring continuously for 7 days (mean 58 years; men: $41 \%$ ), the authors evaluated the association between meteorological factors (outdoor temperature and atmospheric pressure) and circadian BP variability. They compared $\mathrm{BP}$ values for the lowest and highest days with regard to the daily mean outdoor temperature and mean atmospheric pressure. In this study, the mean difference in outdoor temperature between the coldest and warmest days was $5.0^{\circ} \mathrm{C}(P<0.0001)$, and the prewaking morning BP surge, which was defined as the difference between the mean systolic BP during the $2 \mathrm{~h}$ after waking and the average systolic BP during the $2 \mathrm{~h}$ before waking, 


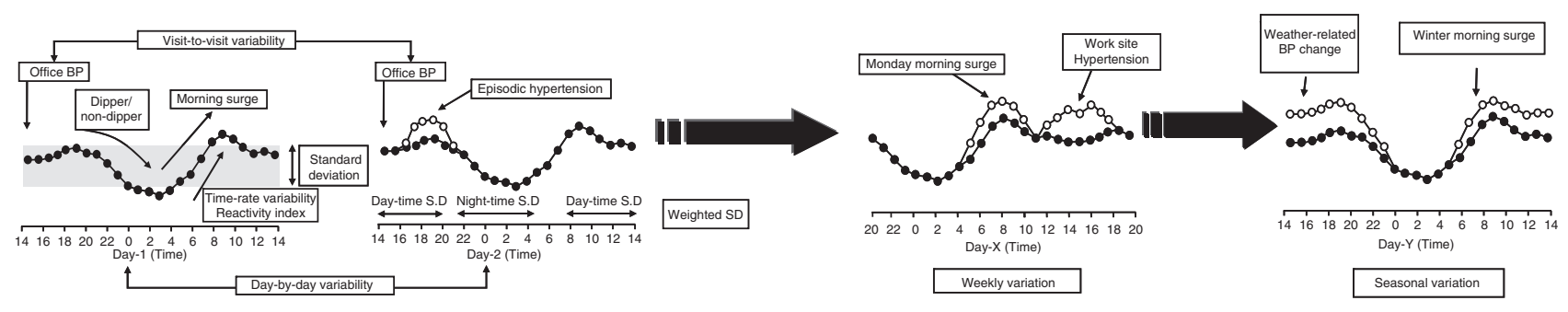

Figure 1 Short-term and long-term blood pressure variations. BP variability consists of at least two types of phenomena: long-term fluctuations, such as daynight, day-to-day, weekly (for example, Monday BP surge) and seasonal changes, and short-term BP variations that occur within the daytime and nighttime periods.

as well as the morning BP level on the coldest day were significantly higher than those on the warmest day (both $P<0.05$ ); on the other hand, there were no significant differences in the levels of awake and sleep BP between the coldest and warmest days. These results were confirmed in a multivariate analysis, in which age and difference in outdoor temperature were significantly and independently associated with prewaking morning BP increase. There have been several reports demonstrating that not only sleep-through MS but also prewaking MS is associated with target-organ damage and CVDs; ${ }^{9,19,20}$ therefore, throughout the winter, and particularly on the coldest days, a specific consideration of morning BP increase and strategy targeting morning BP increase would be required to prevent cardiovascular risks.

The strengths of this study are as follows. First, in contrast to previous reports ${ }^{13,15,16}$ that examined the association between cold temperature and BP levels and BP variability using different subjects examined in different months of the year, the study by Murakami et al. included a single group of subjects who were continuously examined by 24-h ambulatory BP monitoring for 7 days. Therefore, this study would seem to contain less noise in its analysis of the association between cold temperature effects and BP parameters. Second, previous studies have examined the association between cold temperature and BP levels and BP variability by assessing their absolute levels. ${ }^{13,15,16}$ In contrast, the study by Murakami et al. has revealed an association between the difference in outdoor temperature between the coldest and warmest days and the difference of prewaking morning BP increase between the coldest and warmest days. This point deserves to be mentioned, because a recent study demonstrated that an increased risk for CVDs in association with cold temperature was more relevant in the context of an unexpected temperature decrease than in terms of the absolute temperature level itself. $^{21}$
The findings of the study by Murakami et al. should be evaluated against the background of several study limitations. First, in this cross-sectional study, the cold temperature was associated not only with prewaking MS but also with greater nocturnal BP decline, although these two factors are reported to have opposite prognostic value; $^{22}$ therefore, prospective studies will be needed to elucidate the prognostic implications of cold temperature. Second, this study was conducted only during the cold season in Japan (October-April), and thus the association during the hot season remains to be established. The latter point should also be kept in mind, because a recent study has demonstrated that the increased risk for the occurrence of CVDs in association with a decrease in air temperature was similar for winter and summer and was more pronounced in years with higher average temperatures. $^{21}$ Third, no information is available regarding the indoor temperature in the homes where the measurements were conducted. Residential use of air conditioners is widespread in Japan, particularly in Hokkaido, where this study was conducted, and thus the true association between cold temperature and morning BP increase could have been underestimated.

In spite of the methodological difficulties, the study by Murakami et al. reinforces the view that there is an association between cold temperature and morning BP increase in the Japanese population. Information about seasonal trends regarding episodes of increased incidence of CVDs and how these trends are influenced by certain risk factors, including air temperature, might be used as a surrogate to predict onset of CVDs and might be helpful in preparing appropriate warnings during periods of increased CVD risk.

1 Tofler GH, Muller JE. Triggering of acute cardiovascular disease and potential preventive strategies. Circulation 2006; 114: 1863-1872.
2 Kario K. Morning surge and variability in blood pressure: a new therapeutic target? Hypertension 2005; 45 : 485-486.

3 Mancia G, Grassi G. Mechanisms and clinical implications of blood pressure variability. J Cardiovasc Pharmacol 2000; 35: S15-S19.

4 Pickering TG, Eguchi K, Kario K. Masked hypertension: a review. Hypertens Res 2007; 30: 479-488.

5 Rothwell PM. Limitations of the usual bloodpressure hypothesis and importance of variability, instability, and episodic hypertension. Lancet 2010; 375: 938-948.

6 Stolarz-Skrzypek K, Thijs L, Richart T, Li Y, Hansen TW, Boggia J, Kuznetsova T, Kikuya M, Kawecka-Jaszcz K, Staessen JA. Blood pressure variability in relation to outcome in the international database of ambulatory blood pressure in relation to cardiovascular outcome. Hypertens Res 2010; 33: 757-766.

7 Schillaci G, Parati G. Determinants of blood pressure variability in youth: at the roots of hypertension. J Hypertens 2010; 28: 660-664.

8 Muller JE, Tofler GH, Stone PH. Circadian variation and triggers of onset of acute cardiovascular disease. Circulation 1989; 79: 733-743.

9 Kario K, Pickering TG, Umeda Y, Hoshide S, Hoshide Y, Morinari M, Murata M, Kuroda T, Schwartz JE, Shimada $\mathrm{K}$. Morning surge in blood pressure as a predictor of silent and clinical cerebrovascular disease in elderly hypertensives: a prospective study. Circulation 2003; 107: 1401-1406.

10 Kario K, Ishikawa J, Pickering TG, Hoshide S, Eguchi K, Morinari M, Hoshide Y, Kuroda T, Shimada K. Morning hypertension: the strongest independent risk factor for stroke in elderly hypertensive patients. Hypertens Res 2006; 29: 581-587.

11 Kelly-Hayes M, Wolf PA, Kase CS, Brand FN, McGuirk JM, D'Agostino RB. Temporal patterns of stroke onset. The Framingham Study. Stroke 1995; 26: 1343-1347.

12 Hampel R, Breitner S, Rückerl R, Frampton MW, Koenig W, Phipps RP, Wichmann HE, Peters A, Schneider A. Air temperature and inflammatory and coagulation responses in men with coronary or pulmonary disease during the winter season. Occup Environ Med 2010; 67: 408-416.

13 Sun Z. Cardiovascular responses to cold exposure. Front Biosci (Elite Ed) 2010; 2: 495-503.

14 Minami J, Kawano Y, Ishimitsu T, Yoshimi H, Takishita S. Seasonal variations in office, home and $24 \mathrm{~h}$ ambulatory blood pressure in patients with essential hypertension. J Hypertens 1996; 14: 1421-1425.

15 Sega R, Cesana G, Bombelli M, Grassi G, Stella ML, Zanchetti A, Mancia G. Seasonal variations in home and ambulatory blood pressure in the PAMELA population. ressione Arteriose Monitorate E Loro Associazioni. J Hypertens 1998; 16: 1585-1592.

16 Modesti PA, Morabito M, Bertolozzi I, Massetti L, Panci G, Lumachi C, Giglio A, Bilo G, Caldara G, Lonati L, Orlandini S, Maracchi G, Mancia G, Gensini GF, Parati G. Weather-related changes in 24-hour blood pressure profile: effects of age and implications for hypertension management. Hypertension 2006; 47: 155-161. 
17 Kario K. Caution for winter morning surge in blood pressure: a possible link with cardiovascular risk in the elderly. Hypertension 2006; 47: 139-140.

18 Murakami S, Otsuka K, Kono T, Soyama A, Umeda T, Yamamoto N, Morita H, Yamanaka G, Kitaura Y. Impact of outdoor temperature on prewaking morning surge and nocturnal decline in blood pressure in a Japanese population. Hypertens Res 2011; 34: 70-73.
19 Gosse P, Lasserre R, Minifié C, Lemetayer P, Clementy J. Blood pressure surge on rising. J Hypertension 2004; 22: 1113-1118.

20 Metoki H, Ohkubo T, Kikuya M, Asayama K, Obara T, Hashimoto J, Totsune K, Hoshi H, Satoh H, Imai Y. Prognostic significance for stroke of a morning pressor surge and a nocturnal blood pressure decline: the Ohasama study. Hypertension 2006; 47: 149-154.
21 Wolf K, Schneider A, Breitner S, von Klot S, Meisinger C, Cyrys J, Hymer H, Wichmann HE, Peters A, Cooperative Health Research in the Region of Augsburg Study Group. Air temperature and the occurrence of myocardial infarction in Augsburg, Germany. Circulation 2009; 120: 735-742.

22 Yano Y, Kario K. Unresolved issues of the morning blood pressure surge: the next stage of clinical applicability for the morning surge. Hypertens Res 2008; 31: 1491-1494. 